\title{
Intradialytic Massage for Leg Cramps Among Hemodialysis Patients: a Pilot Randomized Controlled Trial
}

\author{
Diane Mastnardo, BS, LMT,* Janice M. Lewis, BA, LMT, \\ Kristi Hall, LMT, Catherine M. Sullivan, MS, RD, LD, \\ Katrice Cain, MA, Jacqueline Theurer, MBA, \\ Anne Huml, MD, Ashwini R. Sehgal, MD \\ Case Western Reserve University and MetroHealth Medical Center, Cleveland, OH, USA
}

Background: Patients on hemodialysis often experience muscle cramps that result in discomfort, shortened treatment times, and inadequate dialysis dose. Cramps have been associated with adversely affecting sleep and health-related quality of life, depression and anxiety. There is limited evidence available about massage in dialysis; however, massage in cancer patients has demonstrated decreases in pain, inflammation, and feelings of anxiety. These correlations indicate massage may be an effective treatment modality for hemodialysis-related lower extremity cramping.

Purpose: To determine the effectiveness of intradialytic massage on the frequency of cramping among hemodialysis patients prone to lower extremity cramping.

Participants: 26 maintenance hemodialysis patients with frequent lower extremity cramps.

Setting: three outpatient hemodialysis centers in Northeast Ohio.

Research Design: randomized controlled trial.

Intervention: The intervention group received a 20-minute massage of the lower extremities during each treatment (three times per week) for two weeks. The control group received usual care by dialysis center staff.

Main Outcome Measure: change in frequency of lower leg cramping.

Results: Patient reported cramping at home decreased by 1.3 episodes per week in the intervention group compared to 0.2 episodes per week in the control group $(p=.005)$. Patient reported cramping during dialysis decreased by 0.8 episodes in the intervention group compared to 0.4 episodes in the control group $(p=0.44)$.

Conclusion: Intradialytic massage appears to be an effective way to address muscle cramping. Larger studies with longer duration should be conducted to further examine this approach.

KEY WORDS : dialysis; massage; muscle cramping

\section{INTRODUCTION}

Over 600,000 Americans receive treatment (most commonly hemodialysis) for permanent kidney failure under Medicare's End-Stage Renal Disease program at an annual cost of $\$ 35$ billion. ${ }^{(1)}$ However, the rapid removal of excess fluid and waste products by hemodialysis contributes to frequent and distressing muscle cramps, typically in the lower extremities. Such cramps, which affect $25 \%-50 \%$ of patients, may occur during hemodialysis treatment or at home following dialysis. ${ }^{(2)}$ Cramps that occur during hemodialysis can lead to shortening of treatment or to reduction of fluid removal which may result in inadequate clearance of waste products, fluid overload, and hypertension. ${ }^{(2,3,4)}$ Shortening of as little as one dialysis treatment per week is associated with a statistically significantly increased mortality risk. ${ }^{(5,6)}$ Cramps also adversely affect sleep and health-related quality of life and are associated with depression and anxiety. ${ }^{(7)}$

The etiology of cramping in dialysis patients is not well known, but several common triggers have been identified including electrolyte abnormalities, hypovolemia, hypotension, dialysis prescription (blood flow rate and ultrafiltration), and dialysate composition. ${ }^{(8,9)}$ Therapeutic maneuvers to manage muscle cramps include intravenous fluids, oral medications, adjustments in dialysis settings, warm moisture compresses, and stretching. ${ }^{(8,9)}$ One pilot study by Ahsan et al. (10) evaluated the feasibility of intradialytic sequential compression devices (SCD) use for the prevention of hemodialysis-related lower extremity cramping. This study found that the application of SCD to lower extremities prevented the generation of hemodialysis related cramping in four cases. Additionally, no adverse side effects were reported. In another study, acupressure with massage in patients with end-stage renal disease (ESRD) who were undergoing dialysis, showed improvement with symptoms of fatigue and depression over12 weeks. ${ }^{(11)}$ Again, no adverse side effects were noted. 
There are no known published research studies on massage for cramping during dialysis, however other manual techniques have had promising results. $(12,13,14,15)$ Massage has increasingly been used in health care over the past 10 years. Research studies using massage in cancer patients have shown a decrease in pain, increase in energy, decreased inflammation and decreased feelings of anxiety when done appropriately. ${ }^{(11)}$ Physiological effects of massage techniques include increasing peripheral circulation, mechanically moving fluid in the veins and lymph, and stimulating the nerves, blood vessels and cell exchange of the tissue being manipulated. ${ }^{(16,17)}$ With the limited evidence available about massage in dialysis patients, it appears that this may be an effective treatment modality for hemodialysis-related lower extremity cramping in carefully selected patients. This pilot research is designed to determine if massage can be utilized as a preventive measure to decrease the frequency of cramping.

\section{METHODS}

The massage therapy protocol was developed by the lead massage therapist based on documented physiological effects of massage. ${ }^{(16,17)}$ Five LMTs were trained using the protocol. They then administered massages as specified in the protocol to three sample participants to help develop intertherapist consistency. Pretesting was done at Centers for Dialysis Care (CDC) West, in Cleveland, Ohio. To assess cramps, we used questionnaires of demonstrated reliability, validity, and sensitivity adapted from the Kidney Disease Quality of Life Short Form (KDQOL-SF). ${ }^{(18)}$ These questions ask patients to report on cramp frequency, location, duration, and severity separately for cramps during dialysis treatment and for cramps that occur at home following dialysis. $(19,20,21)$ We further tested our instruments on a group of three dialysis patients who met all study eligibility criteria. The pretest subjects completed all study tasks as outlined below for the full trial, with the exception of receiving only one massage.

\section{Participants}

The study was conducted utilizing three long-term hemodialysis facilities with approximately 500 total dialysis patients within the Centers for Dialysis Care Network in Cleveland, OH. To determine study eligibility, study coordinators used a chart abstraction to identify patients who had one or more episode of lower extremity cramping during or after dialysis in the previous two weeks. Additional eligibility criteria were age 18-90 years, having received hemodialysis for at least six months, having a stable dry weight for the previous month (less than $2 \mathrm{~kg}$ change), and having the ability to give informed consent. We excluded new patients as the first several months of dialysis treatment is often a time of hemodynamic instability, and excluded patients with a fluctuating dry weight as this fluctuation may cause cramping. We also excluded patients with sores, wounds, amputation, dermatitis, or edema of the lower extremities, with a known history of deep vein thrombosis (DVT) or peripheral vascular disease (PVD), or with a vascular access in a lower extremity.

A research assistant invited eligible hemodialysis patients to participate in the study, informed patients about study procedures, and obtained informed consent at the dialysis facilities while patients were receiving hemodialysis treatment. Consenting patients were randomized into intervention and control groups using a random number generator. All enrolled patients received a $\$ 25$ gift card for participating in the study, and intervention patients receive the massage free of charge. The study was approved by the institutional review board of MetroHealth Medical Center Cleveland, $\mathrm{OH}$.

\section{Intervention Group}

The intervention group received a 20-minute massage by trained licensed massage therapists during each hemodialysis treatment attended for two weeks, as well as usual care. Most patients receive dialysis three times per week, resulting in six massage sessions. Prior to performing a massage, the therapist checked with each patient's dialysis nurse or technician to determine if there were clinical or hemodynamic abnormalities to preclude treatment. These abnormalities included: acute shortness of breath determined by nurse or technician observation or poor oxygen saturation, fever, hypotension, or volume overload ( $>5 \mathrm{~kg}$ above recorded dry weight). In order to avoid postprandial blood flow redistribution, dialysis patients receiving massage treatment for cramping were asked to refrain from eating during the massage session.

The massage included both feet and legs up to and including the knee. Massage included general light centripetal friction, kneading and point compression to bellies, and myotendinous junction of muscles of the foot and calf not to exceed a perceived pain of 6 on a scale of 1 to 10,10 being most severe and 1 being no pain. The Wong-Baker FACES pain rating scale was provided to patients while perceived pain was being explained (see Table 1 for intervention protocol). The massage therapist performed a short evaluation to verify the patient was free from new skin problems on the legs or feet including redness, heat, rashes and sores, blood clots in legs, vascular access in lower extremity, and no hospitalizations since last treatment to be eligible for treatment that day. Post-treatment notes were completed to identify any concerns with the massage. Massage therapists were compensated $\$ 25$ for each massage scheduled. 
Protocol starts with left leg and is completed on the right leg. Routine is performed 2 times on left leg then right leg, to total 20 min.

1. Start with passive touch, cup the patients left heel with left hand, right hand on the posterior calf index finger at bend of knee; $5 \mathrm{sec}$.

2. Place cream/oil on leg with 8 strokes of alternating centripetal friction from distal to proximal (heal to knee) starting with left hand on posterior lower leg. Right hand on top. Left hand thumb on the anterior medial section of calf, 4 fingers on back of calf. Right hand thumb on the anterior lateral section of calf, 4 fingers on posterior of calf; 2 times; $35 \mathrm{sec}$

3. 3 strokes of centripetal friction with hands (palmar surface) together from ankle to knee (thumbs anterior, 4 fingers on back facing each other); $12 \mathrm{sec}$

4. Palmar kneading-alternating hands from ankle to knee compressing and lifting (posterior and lateral aspects of lower leg); 3 times; $18 \mathrm{sec}$

5. 3 strokes of centripetal friction with hands together (palmar surface) from ankle to knee (thumbs anterior, 4 fingers on back facing each other); $12 \mathrm{sec}$

6. Point compression top of knee (medial superior to patellar on tendon), lateral and medial femoral condyles, and below knee (inferior to patella at femoral-tibial junction) hold for $3 \mathrm{sec}$ each; $12 \mathrm{sec}$

7. Point compression at top of calf (proximal attachment of gastrocnemius) right hand lateral, left hand medial hold for $3 \mathrm{sec}$

8. Point compression middle of calf (belly of soleus) hold for $3 \mathrm{sec}$

9. Point compression bottom of calf (musculotendinous junction of gastrocnemious and soleous -Achilles tendon) hold for 3 seconds

10. Palmer compression exerted to the plantar and dorsal surfaces of the foot; $12 \mathrm{sec}$

11. 3 strokes of centripetal friction from toes to ankle left hand on top of foot, right on bottom; $12 \mathrm{sec}$

12. Digital kneading of foot; $12 \mathrm{sec}$

13. 3 strokes of centripetal friction toes to knee (left hand on top of foot, right on bottom, at ankle right hand lateral, left medial thumbs anterior, 4 fingers on back facing each other); $24 \mathrm{sec}$

14. Palmar kneading-alternating hands from ankle to knee compressing and lifting (posterior and lateral aspects of lower leg). 3 times; $18 \mathrm{sec}$

15. 3 strokes of centripetal friction from toes to above knee (left hand on top of foot, right on bottom, at ankle right hand lateral, left medial thumbs anterior, 4 fingers on back facing each other); $24 \mathrm{sec}$

16. Passive range of motion (PROM) of ankle plantar flexion and dorsiflexion 3 times (cup ankle with left hand, slight traction of ankle, and place right heel of hand just below the ball of foot); $12 \mathrm{sec}$

17. PROM ankle 3 circles clockwise, 3 counter clockwise (cup ankle with left hand, slight traction of ankle, and place heel of right hand just below the ball of foot); $18 \mathrm{sec}$

18. PROM plantar flexion and dorsiflexion 3 times (Cup ankle with left hand, slight traction of ankle, and place right hand just below the ball of foot); $12 \mathrm{sec}$

19. 3 strokes of centripetal friction from toes to knee; $24 \mathrm{sec}$.

Total time: 4 min $10 \mathrm{sec}$. Repeat protocol (total 2 times on each leg); extra $50 \mathrm{sec}$ for varying leg size and switching between legs.

\section{Training and Monitoring of Study Staff and Massage Therapists}

During a two-month training period, the lead licensed massage therapist trained five state licensed massage therapists from independent practices throughout the community in intervention massage protocol and assessed for intertherapist consistency. All study massage therapists were trained about the study protocol including data collection, safety assessments, and completed all Institutional Review
Board required training. This entailed certification in protection of human subjects, including proper procedures for recruiting and consenting subjects and the need to maintain confidentiality. Cultural competency training, dialysis treatment, and facility etiquette were also covered.

\section{Control Group}

The control group received usual care by dialysis center staff. Cramps that occur during hemodialysis 
treatment are generally addressed by correcting any hypotension, stopping further fluid removal, stretching the affected muscle, and, if cramps continue, acutely raising plasma osmolality with hypertonic saline or dextrose. Cramps that occur at home following dialysis treatment have not been well studied. Nephrologists typically recommend that patients try one or more of the following for cramps at home: stretching, rubbing the cramp, walking or jiggling the lower extremity, or taking a warm bath. We are reporting according to the CONSORT extension for nonpharmaceutical interventions.

\section{Data Collection}

Research assistants examined the charts from the dialysis facility. Chart abstractions were used to assess eligibility using the inclusion and exclusion criteria, and to obtain demographic and medical characteristics of participants. Intervention and control subjects were interviewed by research assistants at enrollment and at the completion of the study regarding their experiences with cramping and pain, both during dialysis treatment and at home between dialysis treatments. For each interview, participants were asked to report on cramping and pain in the previous two weeks.

\section{Analysis}

We used descriptive statistics (means, standard deviations, percentages) to describe the study participants. For each participant, we calculated change in episodes of cramps as the difference between final and baseline. We used the $t$ test to compare change in cramping among intervention vs. control patients. A two-sided $p$ value $<.05$ was considered statistically significant.

\section{RESULTS}

We recruited 16 intervention participants and 16 control participants. Prior to beginning the trial, we found out that two intervention and one control patient did not meet our eligibility criteria. In addition, three intervention patients were unable to receive four or more scheduled massage treatments, so we excluded them from the data analysis.

Characteristics of the participants that completed the study are presented in Table 2. Intervention and control patients were similar in demographic and medical characteristics. Table 3 identifies the change in cramping from baseline to poststudy period. Patients reported a significant decrease in cramping episodes at home by 1.3 in the intervention group versus 0.2 in the control group $(p=.005)$. Subjects reported a decrease in cramping episodes at dialysis of 0.8 in the intervention group and 0.4 in the control
TABLE 2. Baseline characteristics of intervention and control subjects ${ }^{\mathrm{a}}$

\begin{tabular}{lccc}
\hline & $\begin{array}{c}\text { Intervention } \\
(\mathrm{n}=11)\end{array}$ & $\begin{array}{c}\text { Control } \\
(\mathrm{n}=15)\end{array}$ & $\begin{array}{c}\mathrm{p} \\
\text { value }\end{array}$ \\
\hline Age, mean (SD), years & $55(15.7)$ & $52(8.7)$ & .72 \\
Male & $6(55)$ & $6(40)$ & .46 \\
Female & $5(45)$ & $9(60)$ & \\
Race & & & \\
Black & $11(100)$ & $14(93)$ & .29 \\
White & $0(0)$ & $1(7)$ & \\
Cause of renal failure & & & \\
Hypertension & $5(45)$ & $6(40)$ & \\
Diabetes & $6(55)$ & $4(27)$ & .15 \\
Glomerulonephritis & 0 & $4(27)$ & \\
Etiology uncertain & 0 & $1(7)$ & \\
Time receiving dialysis, mean (SD), & $3.9(2.1)$ & $9.9(8.9)$ & .12 \\
years & & & \\
\hline
\end{tabular}

aData are expressed as number (percentage) of participants unless otherwise indicated.

TABLE 3. Change in cramping from baseline to post study period

\begin{tabular}{ccc}
\hline Intervention & Control & $\mathrm{p}$ \\
$\mathrm{n}=11$ & $\mathrm{n}=15$ & value
\end{tabular}

Total massages received,

mean (SD), $\mathrm{n}$

$\begin{array}{ccc}5.4(0.8) & 0 & \\ 3.2 & 2.5 & 0.05 \\ 2.7 & 2.5 & 1 \\ -2.5 & -0.3 & 0.005 \\ -1.6 & -0.9 & 0.44\end{array}$

Baseline cramping at home

(interview)

Baseline cramping at dialysis

(interview)

Change in cramping at home from

prestudy to poststudy (interview)

Change in cramping at dialysis from prestudy to poststudy (interview)

$-1.6$

group ( $p=0.44)$, although not statistically significant. In general, patients were eager to receive massage treatments, reported uniformly high satisfaction with their massage therapists, and had no adverse effects related to massage.

\section{DISCUSSION \& CONCLUSION}

The results detected a very strong effect $(p=.005)$ for massage to decrease cramps at home following dialysis. Decrease in cramps during dialysis did not reach statistical significance. Cramps at home decreased by about $80 \%$ and during dialysis by about $40 \%$ among patients who received massage. Strengths of our study include well-defined subject selection criteria, a randomized controlled trial design, 
high compliance of subjects to assigned massage treatments, and use of validated outcome measures.

To our knowledge, there have not been any previous studies of massage for dialysis cramps. However, an uncontrolled study of four hemodialysis patients with frequent muscle cramps found that applying a mechanical compression device to the legs reduced the frequency of cramps. ${ }^{(10)}$ A study with 80 participants utilizing reflexology to decrease fatigue, pain, and cramps in hemodialysis patients showed promising results. ${ }^{(13)}$ While promising, our findings must be considered preliminary because of several limitations. The number of participants was small, there was a small difference between the two groups in number of cramps at home, only six massages were provided to each patient, there was no long-term follow-up, the study was conducted in a single geographic area, and outcomes were self-reported.

Further work in several areas is needed to confirm and expand on our study findings for generalizability. First, these findings should be demonstrated in larger and more diverse patient groups in multiple centers with additional therapists. Second, future studies should determine the optimal dosage of massage and identify the mechanism of massage that may be affecting cramping. Third, other outcomes, such as shortening of treatment time, dialysis adequacy, morbidity, mortality, quality of life, and health care costs, should be studied.

\section{ACKNOWLEDGMENTS}

This publication was made possible by the Clinical and Translational Science Collaborative of Cleveland, UL1TR000439 from the National Center for Advancing Translational Sciences (NCATS) component of the National Institutes of Health and NIH roadmap for Medical Research. Its contents are solely the responsibility of the authors and do not necessarily represent the official views of the NIH.

\section{CONFLICT OF INTEREST NOTIFICATION}

The authors declare there are no conflicts of interest.

\section{COPYRIGHT}

Published under the CreativeCommons AttributionNonCommercial-NoDerivs 3.0 License.

\section{REFERENCES}

1. National Institutes of Health [Internet]. USRDS Annual Data Report. Vol.II: Altas of end-stage renal disease in the United
States. Bethesda, MD: NIH; 2013. Available from: http://www. usrds.org/2013/pdf/v2_00_intro_13.pdf

2. Kobrin SM, Berns JS. Quinine - a tonic too bitter for hemodialysis-associated muscle cramps? Sem Dial. 2007;20(5):396-401.

3. Rocco MV, Burkart JM. Prevalence of missed treatments and early sign-offs in hemodialysis patients. J Am Soc Nephrol. 1993;4(5):1178-1183.

4. Daugirdas J, Blake P, Ing T. Handbook of Dialysis. Philadelphia, PA: Lippincott Williams \& Wilkins; 2014.

5. Leggat JE Jr. Psychosocial factors in patients with chronic kidney disease: Adherence with dialysis: a focus on mortality risk. Sem Dial. 2005;18(2):137-141.

6. Leggat JE Jr, Orzol SM, Hulbert-Shearon TE, Golper TA, Jones CA, Held PJ, et al. Noncompliance in hemodialysis: predictors and survival analysis. Am J Kidney Dis. 1998;32(1):139-145.

7. Parfrey PS, Vavasour HM, Henry S, Bullock M, Gault MH. Clinical features and severity of nonspecific symptoms in dialysis patients. Nephron. 1988;50(2):121-128.

8. Holley JL, Bems JS, Sheridan AM. Muscle cramps in dialysis patients. Available on the Up To Date website: $\mathrm{http} / /$ www.uptodate.com/contents/muscle-cramps-in-dialysispatients?source $=$ search_result\&search $=$ dialysis + bems + holley \&selectedTitle $=41 \% 7 \mathrm{E} 150$. Accessed on 27 April 2012.

9. Daugirdas JT, Blake PG, Ing TS. Handbook of Dialysis, 4th edition. Philadelphia, PA: Lippincott Williams and Wilkins; 2007.

10. Ahsan M, Gupta M, Omar I, Frinak S, Gendjar S, Osman-Malik $\mathrm{Y}$, et al. Prevention of hemodialysis-related muscle cramps by intradialytic use of sequential compression devices: a report of four cases. Hemodial Int. 2004;8(3):283-286.

11. Yeh SC, Yeh HF. [Using complementary therapy with a hemodialysis patient with colon cancer and a sense of hopelessness] [in Chinese]. J Nurs (China) 2007;54(5):93-98.

12. Kim DH, Yoon DM, Yoon KB. The effects of myofascial trigger point injections on nocturnal calf cramps. J Am Board Fam Med. 2015;28(1):21-27. doi:10.3122/jabfm.2015.01.140151

13. Ozdemir G, Ovayolu N, Ovayolu O.The effect of reflexology applied on haemodialysis patients with fatigue, pain and cramps. Int J Nurs Pract. 2013;19(3):265-73. doi:10.1111/ ijn. 12066

14. Sabouhi F, Kalani L, Valiani M, Mortazavi M, Bemanian M. Effect of acupressure on fatigue in patients on hemodialysis. Iran J Nurs Midwifery Res. 2013;18(6):429-434.

15. Eğlence R, Karataş N, Taşci S. The effect of acupressure on the level of fatigue in hemodialysis patients. Altern Ther Health Med. 2013;19(6):23-31. PMID: 24254035

16. Goats C., Geoffrey. Massage-the scientific basis of an ancient art: part 2. Physiological and therapeutic effects. BR J Sports Med 1994; 28(3):153-156.

17. Kellogg JH. The Art of Massage. Battle Creek, MI: Modern Medicine Publishing Co.; 1929.

18. Hays RD, Kallich JD, Mapes DL, Coons SJ, Amin N, Carter WB, et al. Kidney Disease Quality of Life Short Form (KDQOL$S F^{T M}$ ), Version 1.3: a Manual for Use and Scoring. Santa Monica, CA: RAND; 1997.

19. Chatrath H, Liangpunsakul S, Ghabril M, Otte J, Chalasani N, Vuppalanchi R. Prevalence and morbidity associated with muscle cramps in patients with cirrhosis. Am J Med. 2012;125(10):1019-1025. 
20. Hays RD, Kallich JD, Mapes DL, Coons SJ, Carter WB. Development of the kidney disease quality of life (KDQOL) instrument. Qual Life Res. 1994;3(5):329-338.

21. Weisbord SD, Fried LF, Arnold RM, Rotondi AJ, Fine MJ, Levenson DJ, et al. Development of a symptom assessment instrument for chronic hemodialysis patients: the Dialysis Symptom Index. J Pain Symptom Manag. 2004;27(3):226-240.
Corresponding author: Diane Mastnardo, BS, LMT, Center for Reducing Health Disparities, MetroHealth Campus of Case Western Reserve University, 2500 MetroHealth Drive, R213, Cleveland, OH 44019, USA

E-mail: dmastnardo@gmail.com 